\title{
Dispersion of Multiwall Carbon Nanotubes in Organic Solvents through Hydrothermal Supercritical Condition
}

\author{
Krishnegowda Jagadish, ${ }^{1}$ Shivanna Srikantaswamy, ${ }^{1}$ Kullaiah Byrappa, \\ Lingaraju Shruthi, ${ }^{2}$ and Mavinakere Ramesh Abhilash ${ }^{2}$ \\ ${ }^{1}$ Centre for Materials Science and Technology, Vijnana Bhavan, University of Mysore, Manasagangotri, Mysore 570006, India \\ ${ }^{2}$ Department of Studies in Environmental Science, University of Mysore, Manasagangotri, Mysore 570006, India
}

Correspondence should be addressed to Shivanna Srikantaswamy; srikantas@hotmail.com

Received 6 May 2015; Revised 28 July 2015; Accepted 30 August 2015

Academic Editor: Xiaosheng Fang

Copyright (C) 2015 Krishnegowda Jagadish et al. This is an open access article distributed under the Creative Commons Attribution License, which permits unrestricted use, distribution, and reproduction in any medium, provided the original work is properly cited.

\begin{abstract}
Multiwall carbon nanotube (MWCNT) composite materials require careful formulation of processing methods to ultimately realize the desired properties. Until now, controlled dispersion of MWCNT remains a challenge, due to strong van der Waals binding energies associated with the MWCNT aggregates. In the present study, an effort has been made to disperse MWCNTs in organic solvents like dichloromethane, ethanol, isopropyl alcohol, and hexane through hydrothermal reaction. Dichloromethane is considered the best solvent for the dispersion of MWCNTs. The characterizations were carried out to find the dispersion design, particle size, and stabilization, which clearly indicate that the desired properties of MWCNTs have been achieved.
\end{abstract}

\section{Introduction}

The finding, synthesis, and characterization of different nanocarbon like fullerenes, nanotubes, and related carbon crystals have received much attention owing to their unique structure and properties $[1,2]$. Among the carbon materials, carbon nanotubes (CNTs) are having more interest both in science and technology. The CNTs are of two types, such as single-wall carbon nanotubes (SWCNTs) and multiwall carbon nanotubes (MWCNTs). SWCNTs are rolled up of single graphene sheet and MWCNTs were rolled up of multiple graphene sheets [3]. Both SWCNTs and MWCNTs have high aspect ratios because of their great micrometer lengths with their nanometer level diameter [4]. Further, the fine dispersion of MWCNTs can overcome the low aspect ratio [5].

The high aspect ratio and strong van der Waals interactions result in entangled and bundled MWCNTs $[6,7]$. Thus, dispersion of MWCNTs is a challenging task for their utilization in nanoscale device applications [8]. The poor dispersion of MWCNTs in organic solvents and water has largely restricted their applications [9]. In organic solvents [10] and polymers [11], the MWCNTs were dispersed in greater prospective applications in the nanoscience.
The process of CNT dispersion requires clean synthesis routes with reduced energy input by less severe conditions [12]. A new process has to be technologically friendly; hydrothermal routes may lead to a reproducible fabrication method of carbon materials. Hydrothermal processing is a heterogeneous reaction in the presence of aqueous solvents or mineralizers under high pressure and temperature conditions to dissolve and recrystallize materials that are relatively insoluble under ordinary conditions [13]. Supercritical fluids (SCFs) are the better reaction medium for hydrothermal processing of nanoparticles with higher reaction rates and smaller particles and the reaction products are stable [13]. The present author had earlier synthesized carbon nanocells and nanotubes using amorphous carbon with hydrothermal reaction [14]. In the present study, an attempt has been made to disperse MWCNTs in different organic solvents through hydrothermal reactions in respective supercritical conditions of solvents. SCFs have greater diffusivity in solid materials and make mass transformations from higher to lower concentration [13]. The resulting materials were sonicated and characterized by Transmission Electron Microscopy (TEM), Raman spectroscopy, UV-vis spectroscopy, and Dynamic 
TABLE 1: Critical temperature of organic solvents.

\begin{tabular}{lc}
\hline Solvents & Critical temperature $\left({ }^{\circ} \mathrm{C}\right)$ \\
\hline Dichloromethane & 237 \\
Ethanol & 243 \\
Isopropyl alcohol & 235 \\
Hexane & 235 \\
\hline
\end{tabular}

Light Scattering (DLS) to understand the degree of dispersion.

\section{Materials and Method}

In this study, MWCNTs (outer diameter 10-20 nm, length 10$30 \mu \mathrm{m}, 95 \%$ assay) were obtained from Sisco Research Laboratory, India. The solvents like dichloromethane, ethanol, isopropyl alcohol, and hexane were obtained from Fisher Scientific, India. The experiments were carried out by using MWCNTs mixed with different organic solvents in the concentration of $80 \mathrm{mg} / \mathrm{L}$. The different organic solvents used for dispersion were dichloromethane, ethanol, isopropyl alcohol, and hexane. The individual solutions along with MWCNTs were placed in ultrasonicator (Sidilu, $20 \mathrm{kHz}$, $300 \mathrm{kV}$ ) and sonicated for $15 \mathrm{~min}$. Later, $20 \mathrm{~mL}$ of the sonicated solutions was placed in Teflon liner with Morey type autoclaves. The autoclaves were heated constantly using a hot air oven at critical temperature of individual solvents (Table 1) [15] for five hours. Subsequently, after five hours, the autoclaves were removed from the oven and cooled down to room temperature. The resultant materials in liner with the solvent were collected carefully in a beaker. They were later sonicated for further dispersion and centrifuged (KEMI, 159 Watts/HP, $230 \mathrm{~V}, 50 \mathrm{~Hz}$ ) at $10000 \mathrm{rpm}$ for 15 minutes to check the stability, because during centrifugation the bundled MWCNTs get settled down and supernatant contains finely dispersed MWCNTs (at lower concentration) [9]. These resultant supernatant solutions containing finely dispersed MWCNTS were used for further characterization.

The dispersions of MWCNTs in different organic solvents were characterized using UV-vis spectrophotometer (Shimadzu UV-1800, optical cell length $10 \mathrm{~mm}$, cell volume $3 \mathrm{~mL}$ ) operated in the range $200-1200 \mathrm{~nm}$ to understand the dispersion of MWCNTs. Baseline correction was carried out using pure solvents and the absorbance values were getting subtracted from dispersed MWCNTs absorbance values [11]. Then, these baseline corrections were subtracted every time with new samples of corresponding solvents.

Later, the MWCNTs dispersions were analyzed using TEM (Hitachi H-7500). A drop of $15 \mu \mathrm{L}$ of the dispersed solution was dropped onto carbon coated TEM grids (300 mesh, $3 \mathrm{~mm}$ ) and viewed under microscope [11]. This study revealed the size of the individual MWCNTs.

The dispersed MWCNTs were analyzed using DLS (Microtrac-nanotrac wave-w 3231 Instruments Ltd., at a fixed scattering angle of 90 at room temperature) by measuring the average particle size. The background correction was taken by pure solvents and then the particle sizes with zeta potential of dispersed MWCNTs were measured [16]. Raman spectroscopy is a convenient, powerful, and nondestructive technique for the characterization of carbon and carbonbased materials like carbon black, carbon nanotubes, and so forth and it has become an important tool for understanding many fundamental aspects of all $\mathrm{sp}^{2}$ carbon systems $[17,18]$. The presence of disorder in $\mathrm{sp}^{2}$-hybridized carbon systems, diameter, and the effect of tube-tube interactions on the vibrational modes have been assessed using Raman spectroscopy [19-21].

\section{Results and Discussion}

The dispersed and individual CNTs are active in the UV-vis region by exhibiting characteristics bands between 200 and $1200 \mathrm{~nm}$, but the bundled CNTs are not active in the same wavelength region [22]. Thus, it is possible to characterize dispersed CNTs by UV-vis spectroscopy. Figure 1 depicts the UV-vis spectra of dispersed MWCNTs obtained through hydrothermal reactions in supercritical conditions of respective organic solvents and standard (untreated) MWCNTs. The absorbance of dispersed MWCNTs was investigated for raw solutions (before centrifugation) and supernatant solutions (after centrifugation) in different organic solvents. Initially, the absorbance of MWCNTs was recorded for the raw solutions and then for the supernatant solutions. The absorbance values (Figure 1) of raw solutions were high when compared to supernatant solutions because of the changes in concentration of solutions [23]. The absorbance of MWCNTs solutions shows a maximum range between 200 and $300 \mathrm{~nm}$ and gradually decreases from UV to near IR, which is partly due to scattering, especially in the lower wavelength range [24]. The supernatant solution of dichloromethane shows maximum absorbance (Figure 1(a)) by UV-vis spectroscopy, which indicates the presence of a good number of dispersed MWCNTs with stable product after centrifugation.

DLS is the most useful technique for characterizing nanomaterials in solutions, because of the Brownian motion of particles. The particle sizes of dispersed MWCNTs were analyzed by using DLS. The untreated MWCNTs have average particle size of $512 \mathrm{~nm}$ but the MWCNTs dispersed in dichloromethane have average particle size (cumulant average) of $38 \mathrm{~nm}$ (Figure 2(a)) which has disentangled from aggregation. The average particle size found in ethanol solution was $143 \mathrm{~nm}$. The isopropyl alcohol and hexane solutions show particle size of 207.7 and $356 \mathrm{~nm}$, respectively, by indicating slightly dispersed nanotube particles, and also these results correlate with TEM analysis (Figures 3(a) to 3(j)) corresponding to dispersion and agglomeration.

Zeta potential was calculated using DLS, which explains the stability of CNTs in solutions by having absolute value greater than $30 \mathrm{mV}$ [25]. The solutions of MWCNTs such as dichloromethane, ethanol, isopropyl alcohol, and hexane show zeta potential values of $31,27.9,24.8$, and $13.5 \mathrm{mV}$, respectively. The zeta potential absolute value $31 \mathrm{mV}$ of solution of the dichloromethane solution resembles higher stability and indicates that nanotubes were dispersed individually without aggregation when compared to others.

The dispersion conditions of MWCNTs were visualized using TEM analysis. Figure 3 shows TEM images of untreated 


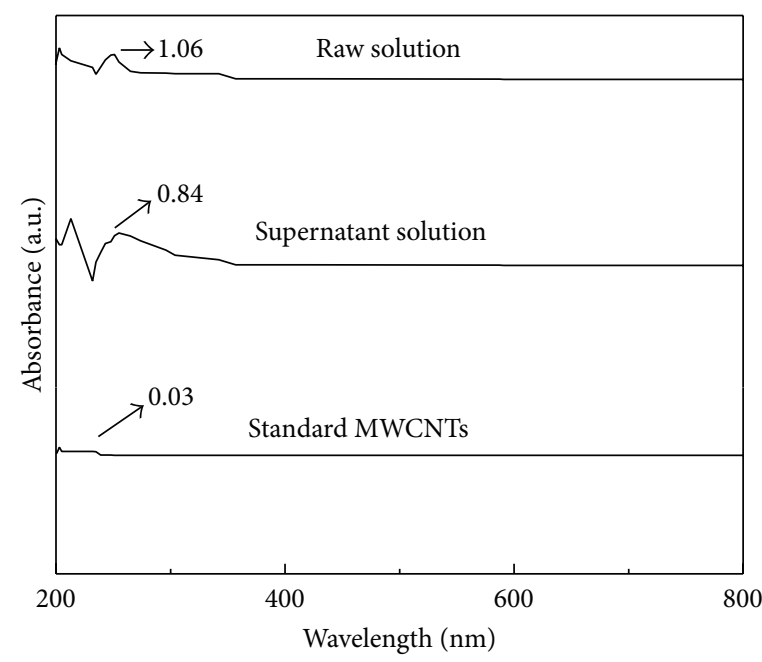

(a)

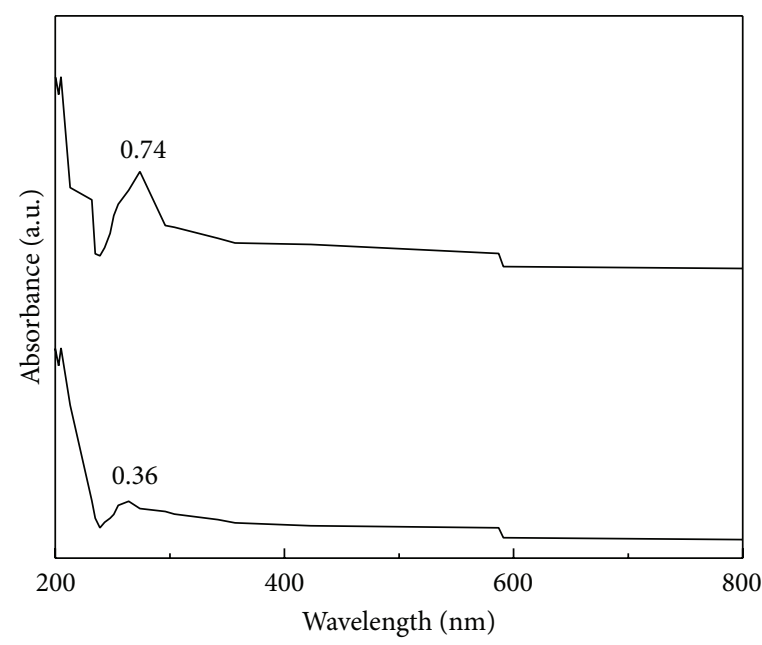

(c)

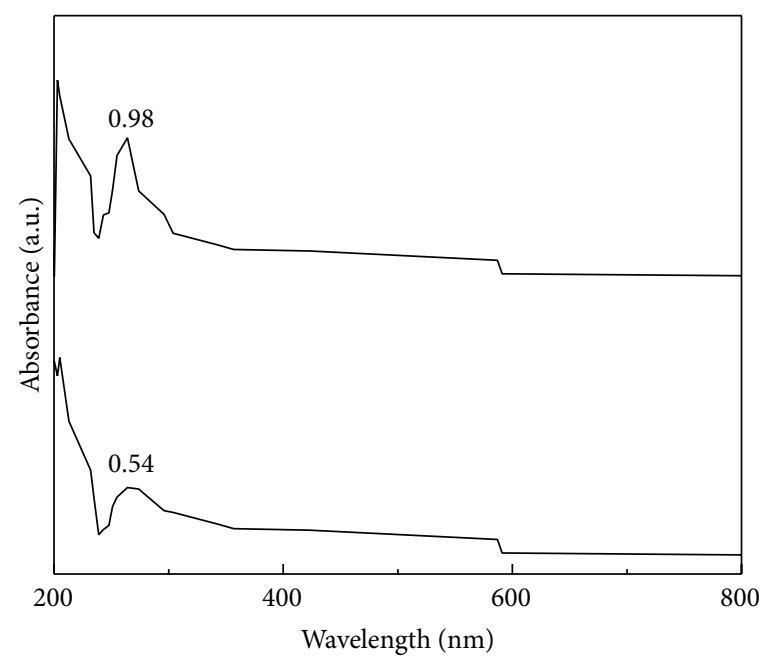

(b)

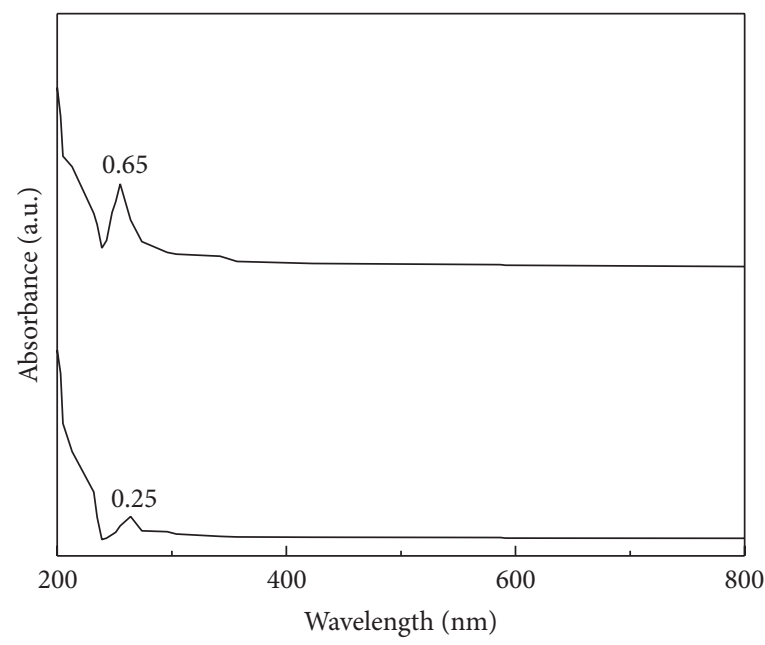

(d)

FIgURE 1: UV-visible spectra of dispersed MWCNTs in (a) dichloromethane and standard (untreated) MWCNTs, (b) ethanol, (c) isopropyl alcohol, and (d) hexane.

MWCNTs and dispersed MWCNTs dispersed in different organic solvents such as dichloromethane, ethanol, isopropyl alcohol, and hexane. The organic solutions containing dispersed MWCNTs (after hydrothermal reactions) were ultrasonicated for $15 \mathrm{~min}$ before TEM analyses to get good images. Based on the TEM image of Figure 3(a), it can be described that the MWCNTs dispersed in dichloromethane have diameter of about 5-10 nm and reduced bundle sizes. Figure 3(b) shows that MWCNTs were disentangled in dichloromethane during hydrothermal reaction under supercritical condition resulting in maximum dispersion. With a very small volume of sample explored in TEM analysis, hence numerous images are required to measure MWCNTs dispersion and bundled sizes.

The TEM study reveals the dispersion and diameter of MWCNTs. Figure 3(c) shows that MWCNTs which were dispersed in ethanol by hydrothermal reaction having outside diameters of about 6-10 and a smaller bundled size were observed (Figures 3(c) and 3(d)). The MWCNTs dispersion in isopropyl alcohol was carried out and the high resolution TEM image (Figures 3(e) and 3(f)) clearly indicated that the diameter was 20 to $50 \mathrm{~nm}$, with slightly dispersed tubes. The maximum aggregation of MWCNTs was observed by TEM analysis in hexane solvent with outer diameters of about $50 \mathrm{~nm}$ (Figures $3(\mathrm{~g})$ and $3(\mathrm{~h})$ ).

Figure 4 shows the Raman spectroscopy of dispersed MWCNTs in different organic solvents. The Raman spectra of untreated MWCNTs are compared to those of dispersed MWCNTs in different organic solvents to evaluate the separation of nanotube bundles. The Raman spectroscopy of untreated MWCNTs (Figure 4(s)) has shown two typical bands at $\sim 1585$ and $\sim 1346 \mathrm{~cm}^{-1}$ which clearly indicate plane vibration of $\mathrm{C}-\mathrm{C}$ bond ( $\mathrm{G}$ band) and disorder band (D band), respectively. Both $D$ band and $G$ band of treated MWCNTs (Figure 4((a), (b), (c), and (d))) have been shifted towards higher wavenumber, which indicates debundling of 


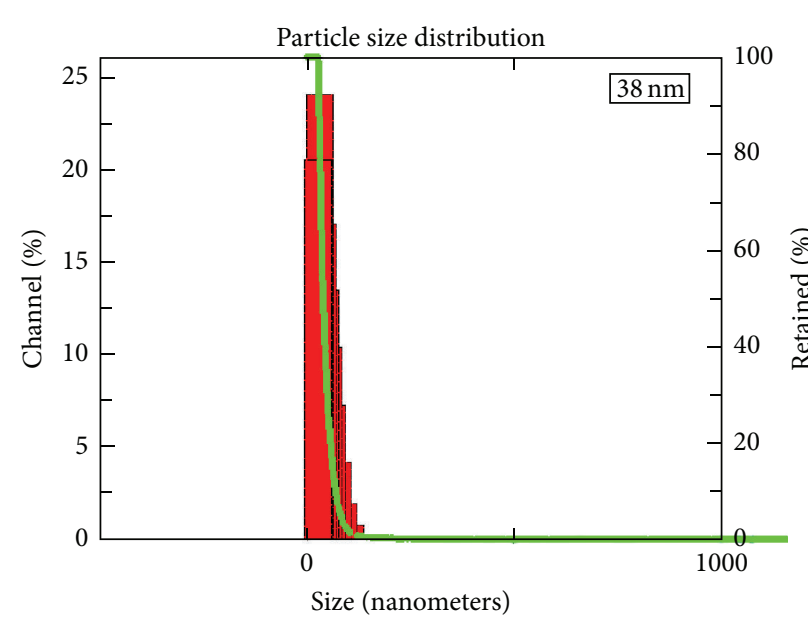

(a)

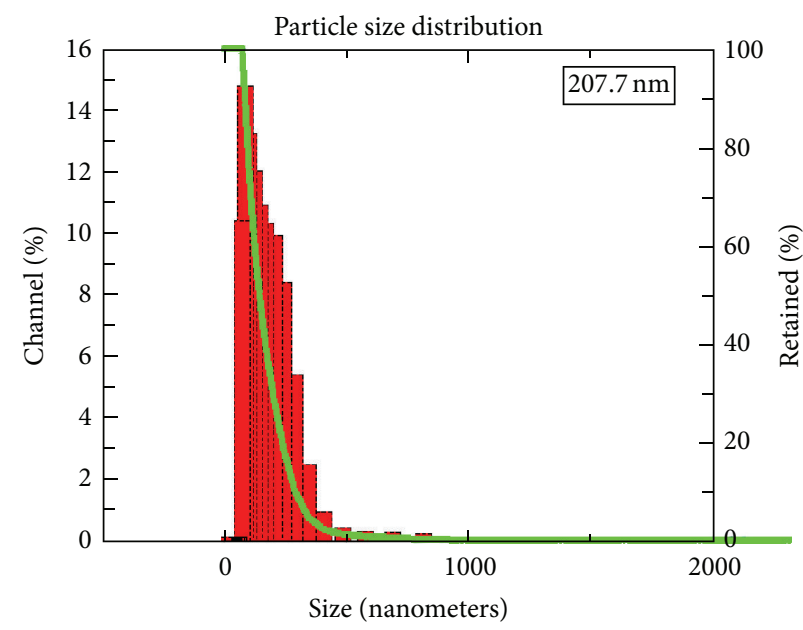

(c)

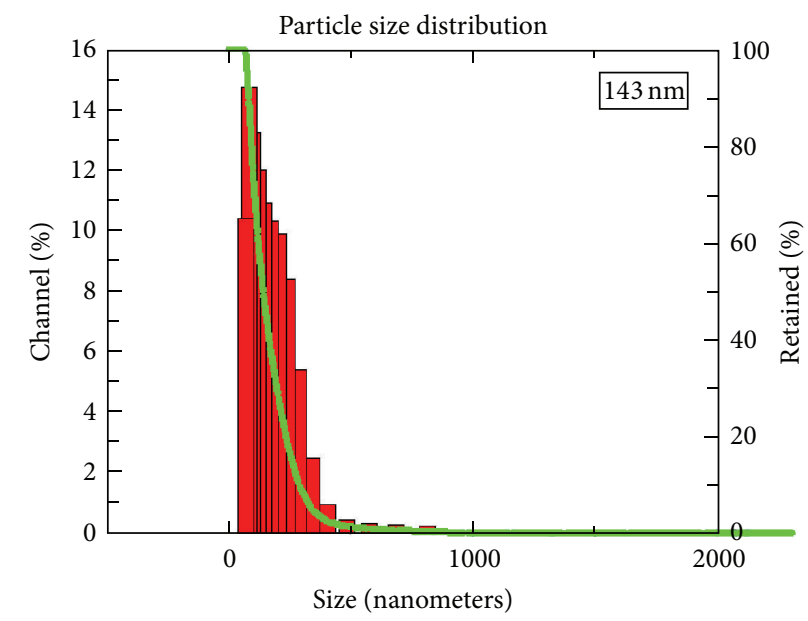

(b)

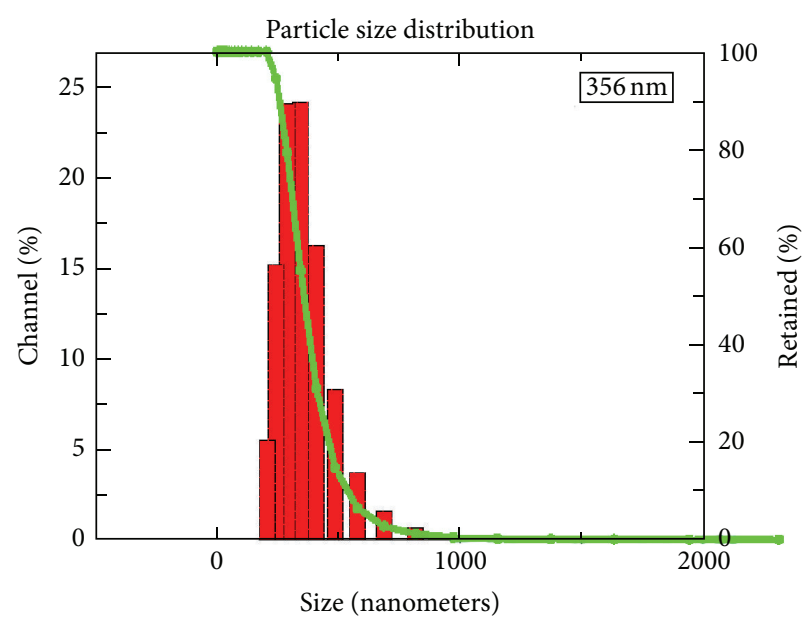

(d)

FIGURE 2: Particle size measurement of dispersed MWCNTs using DLS in (a) dichloromethane, (b) ethanol, (c) isopropyl alcohol, and (d) hexane.

the tubes [26]. The ratio between D and G band has not been changed, which also indicates that the tube diameter has not been changed in hydrothermal reaction. Among all the solutions, the debundling and dispersion of MWCNTs in dichloromethane are more with higher wavenumber shifting from 1346 to $1354 \mathrm{~cm}^{-1}$ and 1585 to $1594 \mathrm{~cm}^{-1}$, of D and $\mathrm{G}$ bands, respectively. The Raman spectroscopy studies are also suited with the other characterization like TEM, UV-vis spectroscopy, and DLS.

\section{Conclusion}

Dispersion of MWCNTs in organic solvents is more applicable and necessary in the science field. The attentiveness of dispersion of MWCNTs in hydrothermal technique is a pleasant task. Different solvents have shown different properties under their supercritical condition. The solvents become fluid and have greater diffusivity in MWCNTs with mass transformations at supercritical condition under hydrothermal reaction. But the properties like diffusivity and mass transformation are not possible at room temperature or at ordinary conditions. Therefore, the choice of the dispersion of MWCNTs in hydrothermal reaction of solvents in supercritical condition shows good results.

The UV-vis region at wavelength 200 to $1200 \mathrm{~nm}$ absorption reveals that the MWCNTS are present as individual and not bundled MWCNTs. The raw MWCNTs and supernatant solutions of centrifuged MWCNTs absorbance indicate the stability of dispersed solutions. DLS study reveals the particle size of MWCNTs and the average sizes of dispersed MWCNTs have clearly explained the very small particle sizes which signify the good dispersion with the support of absolute zeta potential value. The morphological analysis of MWCNTs using TEM is a significant method, but the small quantity of sample during analysis indicates the necessity of a number of images per sample. In the present study, TEM analysis of MWCNTS dispersed in different solutions shows sizes of 5 to $50 \mathrm{~nm}$ diameter. All the above characterization techniques were correlated with the Raman analysis of hydrothermally dispersed MWCNTs; Raman spectroscopy 


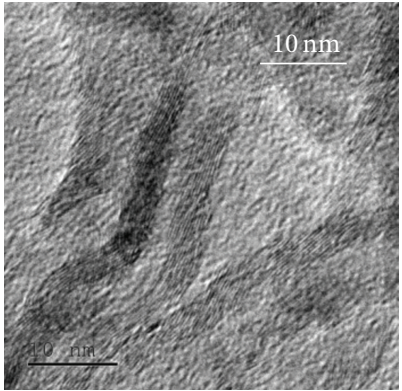

(a)

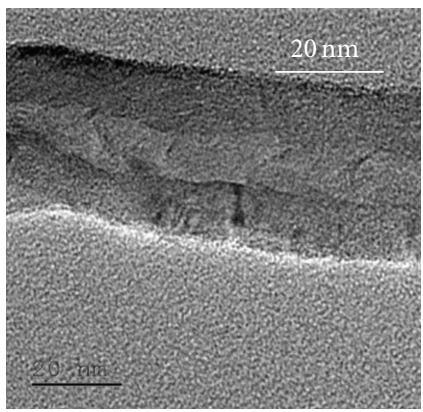

(e)

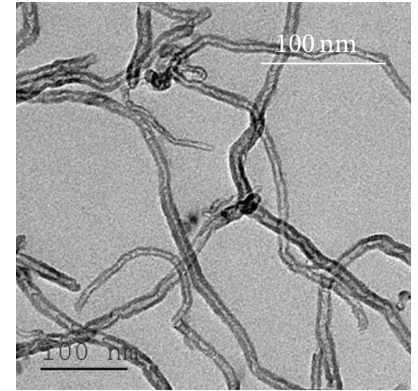

(b)

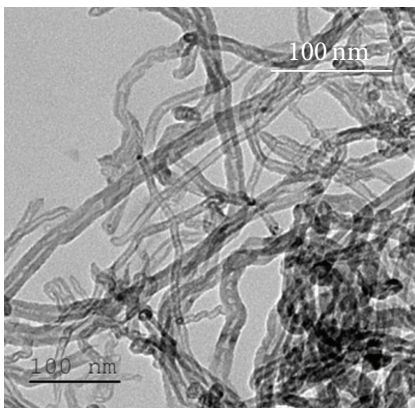

(f)

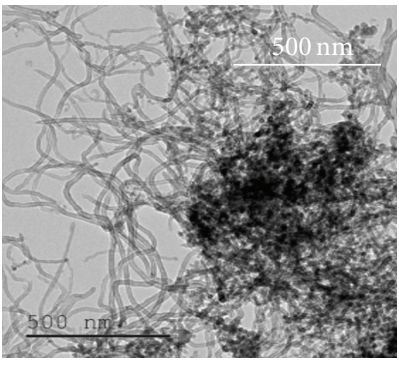

(i)

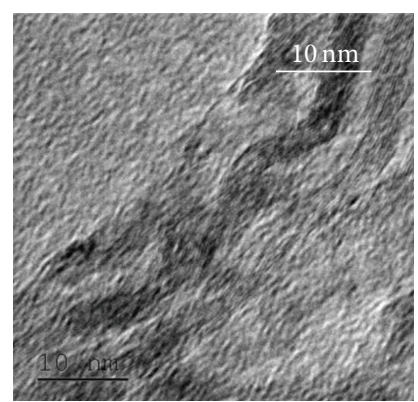

(c)

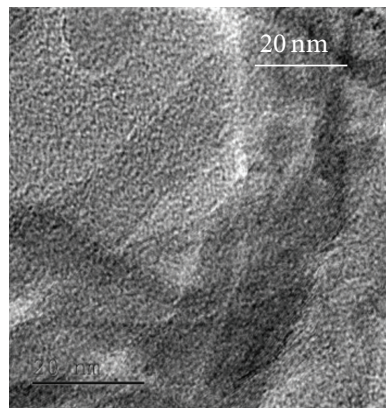

(g)

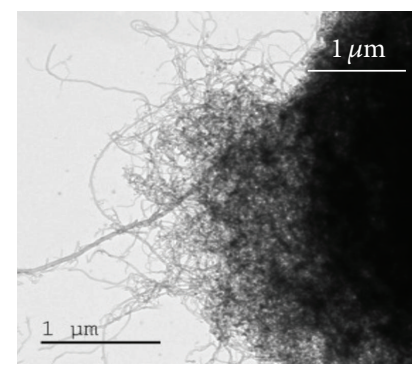

(j)

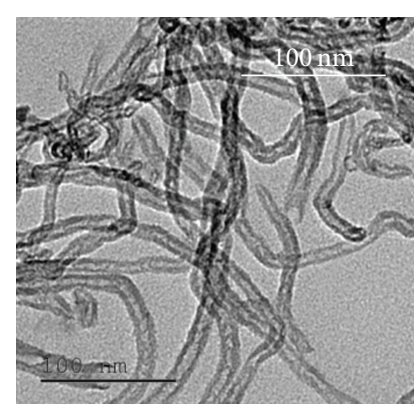

(d)

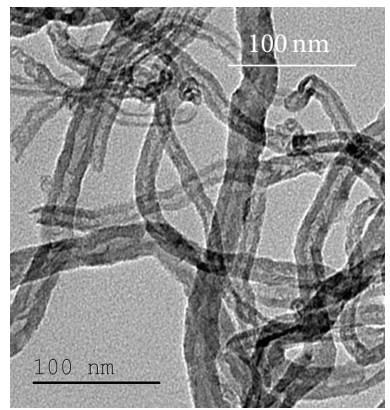

(h)

Figure 3: Transmission electron microscope images of dispersed MWCNTs in dichloromethane (a, b), ethanol (c, d), isopropyl alcohol, (e, f) hexane (g, h), and standard (untreated) MWCNTs.

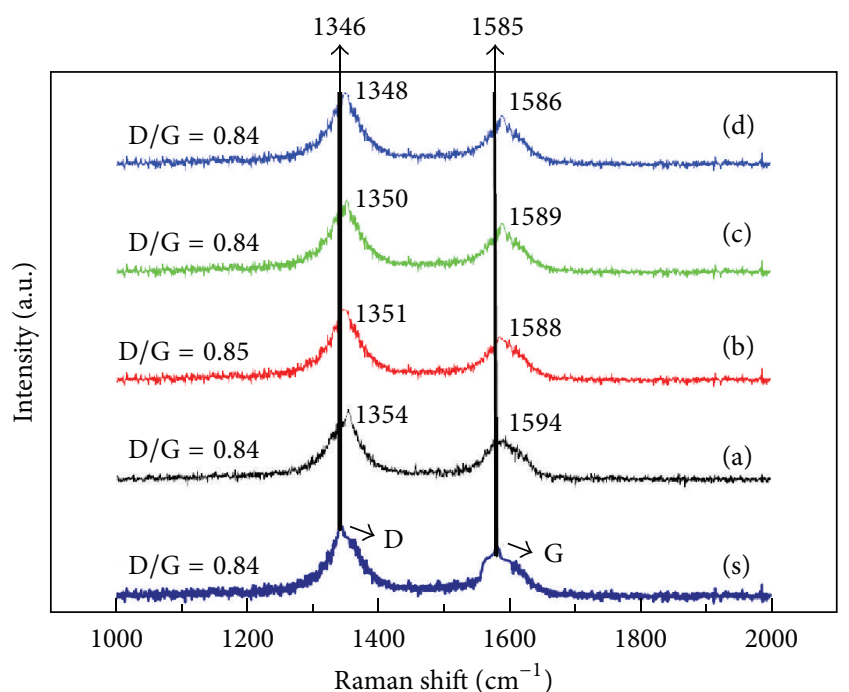

Figure 4: Raman spectroscopy of dispersed MWCNTs in (s) standard MWCNTs, (a) dichloromethane, (b) ethanol, (c) isopropyl alcohol, and (d) hexane. revealed debundling by shifting of $\mathrm{G}$ and $\mathrm{D}$ band and also the MWCNTs diameter has not been changed.

\section{Conflict of Interests}

The authors declare that there is no conflict of interests regarding the publication of this paper.

\section{Acknowledgments}

The authors are grateful to acknowledge the UGC, New Delhi, for supporting this research work under Centre with Potential for Excellence in Particular Area (CPEPA) and University with Potential for Excellence (UPE) for the funding of this research program.

\section{References}

[1] H. W. Kroto, J. R. Heath, S. C. O'Brien, R. F. Curl, and R. E. Smalley, "C60: Buckminsterfullerene," Nature, vol. 318, no. 6042, pp. 162-163, 1985. 
[2] S. Iijima, "Helical microtubules of graphitic carbon," Nature, vol. 354, no. 6348, pp. 56-58, 1991.

[3] K. Li, G. Eres, J. Howe et al., "Self-assembly of graphene on carbon nanotube surfaces," Scientific Reports, vol. 3, article 2353, 2013.

[4] M. Zhang and J. Li, "Carbon nanotube in different shapes," Materials Today, vol. 12, no. 6, pp. 12-18, 2009.

[5] K. S. Park and J. R. Youn, "Dispersion and aspect ratio of carbon nanotubes in aqueous suspension and their relationship with electrical resistivity of carbon nanotube filled polymer composites," Carbon, vol. 50, no. 6, pp. 2322-2330, 2012.

[6] M. S. Dresselhaus, G. Dresselhaus, and P. Avouris, Eds., Carbon Nanotubes: Synthesis, Structure, Properties, and Applications, Springer, 2000.

[7] X. Huang, W. Liang, and S. Zhang, "Radial corrugations of multi-walled carbon nanotubes driven by inter-wall nonbonding interactions," Nanoscale Research Letters, vol. 6, no. 53, pp. 1-6, 2011.

[8] R. Vajtai, Ed., Springer Handbook of Nanomaterials, SpringerVerlag, New York, NY, USA, 2013.

[9] J. Yu, N. Grossiord, C. E. Koning, and J. Loos, "Controlling the dispersion of multi-wall carbon nanotubes in aqueous surfactant solution," Carbon, vol. 45, no. 3, pp. 618-623, 2007.

[10] Z. Hui, Z. Y. Wang, J. U. Mei et al., "Enhanced dispersion of nanotubes in organic solvents by donor-acceptor interaction between functionalized poly(phenylacetylene) chains and carbon nanotube walls," Journal of Polymer Science, Part A: Polymer Chemistry, vol. 47, no. 19, pp. 4995-5005, 2009.

[11] Y. Y. Huang and E. M. Terentjev, "Dispersion and rheology of carbon nanotubes in polymers," International Journal of Material Forming, vol. 1, no. 2, pp. 63-74, 2008.

[12] J. M. Calderon-Moreno, S. S. Swamy, and M. Yoshimura, "Evolution of single-wall carbon nanotubes during hydrothermal treatment," Solid State Ionics, vol. 151, no. 1-4, pp. 205-211, 2002.

[13] K. Byrappa and T. Adschiri, "Hydrothermal technology for nanotechnology," Progress in Crystal Growth and Characterization of Materials, vol. 53, no. 2, pp. 117-166, 2007.

[14] J. M. Calderon Moreno, S. S. Swamy, T. Fujino, and M. Yoshimura, "Carbon nanocells and nanotubes grown in hydrothermal fluids," Chemical Physics Letters, vol. 329, no. 34, pp. 317-322, 2000.

[15] M. I. Smallwood, Handbook of Organic Solvent Properties, Arnold Publishers, London, UK; John Wiley \& Sons, New York, NY, USA, 1996.

[16] X. Cheng, J. Zhong, J. Meng et al., "Characterization of multiwalled carbon nanotubes dispersing in water and association with biological effects," Journal of Nanomaterials, vol. 2011, Article ID 938491, 12 pages, 2011.

[17] M. S. Dresselhaus, A. Jorio, M. Hofmann, G. Dresselhaus, and R. Saito, "Perspectives on carbon nanotubes and graphene Raman spectroscopy," Nano Letters, vol. 10, no. 3, pp. 751-758, 2010.

[18] A. Jorio, M. A. Pimenta, A. G. Souza Filho, R. Saito, G. Dresselhaus, and M. S. Dresselhaus, "Characterizing carbon nanotube samples with resonance Raman scattering," New Journal of Physics, vol. 5, no. 1, article 139, 7 pages, 2003.

[19] R. Graupner, "Raman spectroscopy of covalently functionalized single-wall carbon nanotubes," Journal of Raman Spectroscopy, vol. 38, no. 6, pp. 673-683, 2007.

[20] A. Cuesta, P. Dhamelincourt, J. Laureyns, A. Martínez-Alonso, and J. M. D. Tascón, "Raman microprobe studies on carbon materials," Carbon, vol. 32, no. 8, pp. 1523-1532, 1994.
[21] A. M. Rao, J. Chen, E. Richter et al., "Effect of van der Waals interactions on the Raman modes in single walled carbon nanotubes," Physical Review Letters, vol. 86, no. 17, pp. 38953898, 2001.

[22] Y. Bai, I. S. Park, S. J. Lee, P. S. Wen, T. S. Bae, and M. H. Lee, "Effect of AOT-assisted multi-walled carbon nanotubes on antibacterial activity," Colloids and Surfaces B: Biointerfaces, vol. 89, no. 1, pp. 101-107, 2012.

[23] S. Attal, R. Thiruvengadathan, and O. Regev, "Determination of the concentration of single-walled carbon nanotubes in aqueous dispersions using UV-visible absorption spectroscopy," Analytical Chemistry, vol. 78, no. 23, pp. 8098-8104, 2006.

[24] D. Roy, N. Tiwari, K. Mukhopadhyay, and A. K. Saxena, "Immobilization of individual nanotubes in graphitic layers for electrical characterization," Nanotechnology, vol. 25, no. 11, Article ID 115701, 2014.

[25] C. Liu and J. Choi, "Improved dispersion of carbon nanotubes in polymers at high concentrations," Nanomaterials, vol. 2, no. 4, pp. 329-347, 2012.

[26] L. Bokobza and J. Zhang, "Raman spectroscopic characterization of multiwall carbon nanotubes and of composites," eXPRESS Polymer Letters, vol. 6, no. 7, pp. 601-608, 2012. 

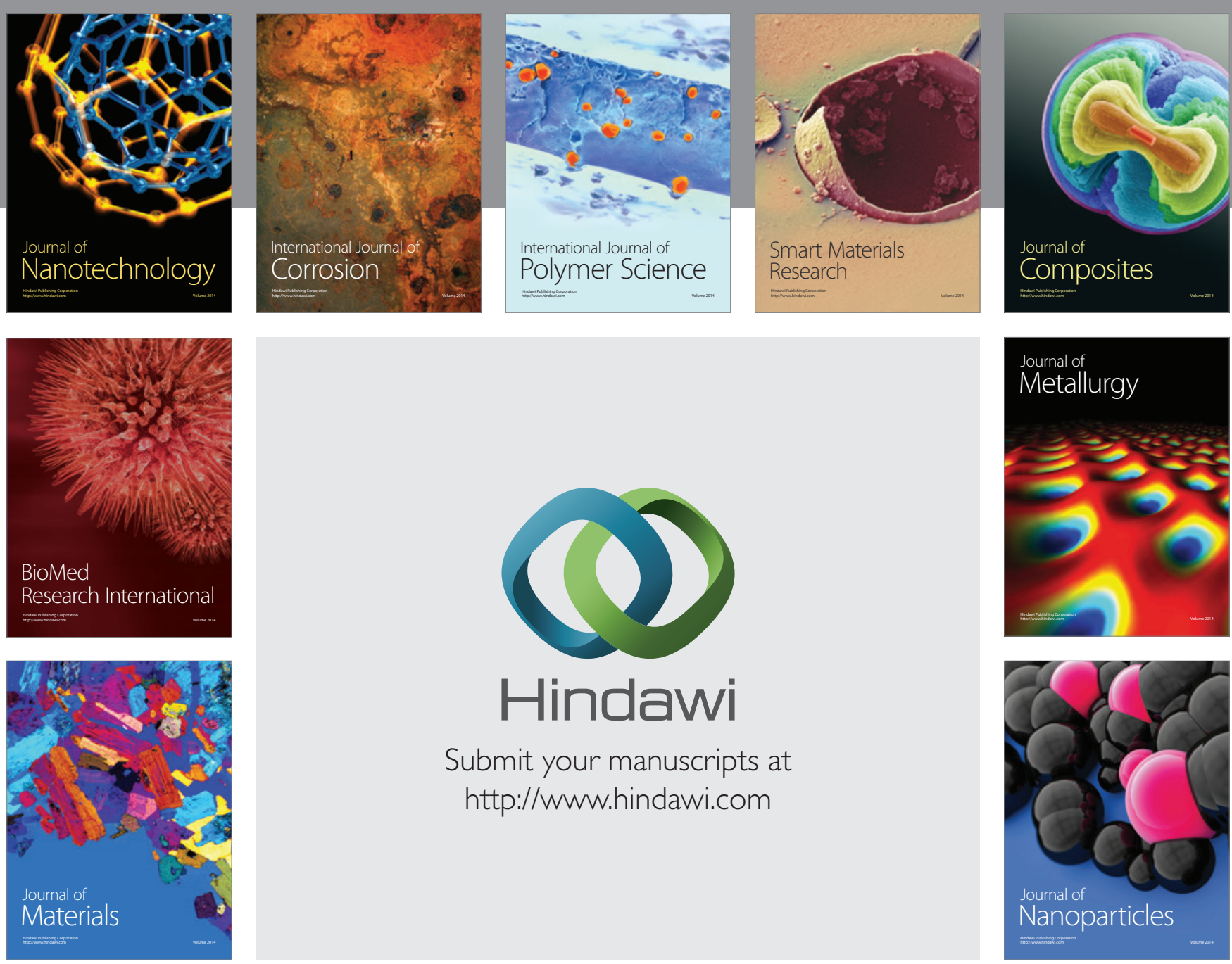

Submit your manuscripts at http://www.hindawi.com
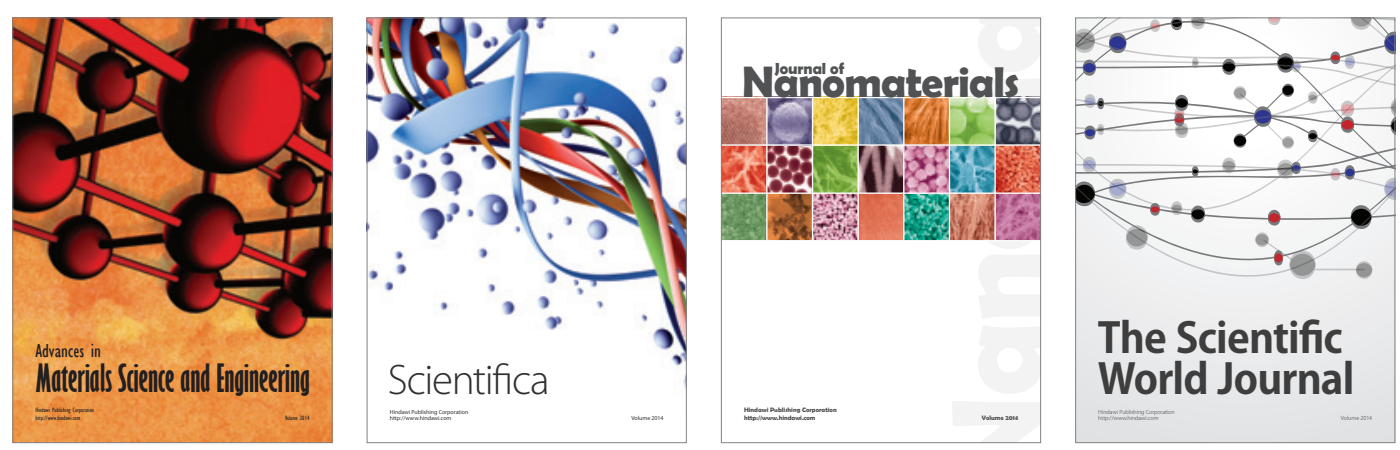

\section{The Scientific World Journal}
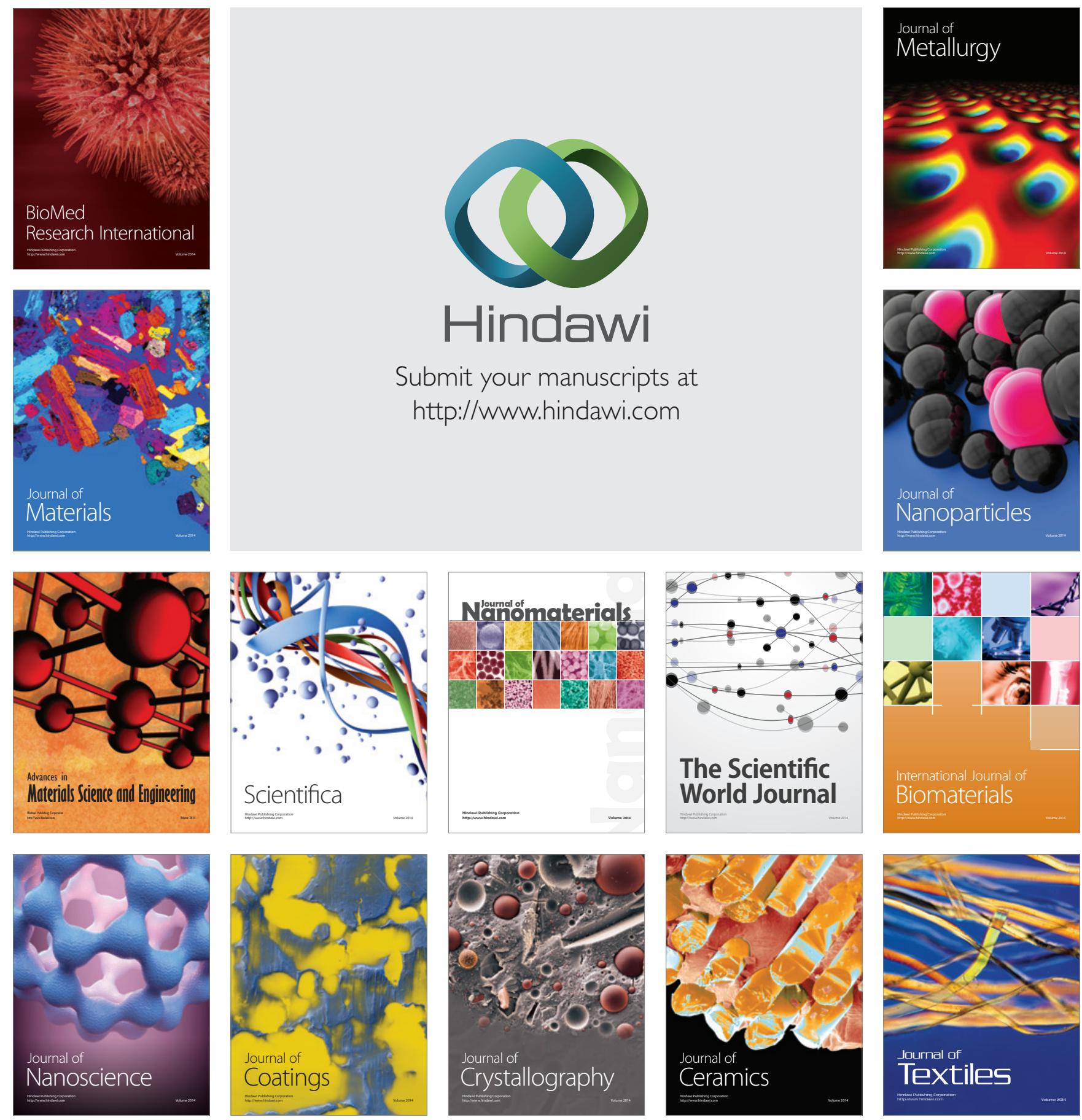